\title{
The effect of cushioning system on impact attenuation of athletic footwear
}

\author{
Stergios Maropoulos ${ }^{1, *}$, Giannis Korakidis ${ }^{1}$, Dimitris Fasnakis $^{1}$, Sophia Papanikolaou ${ }^{1}$, \\ Maria Papagiannaki ${ }^{2}$, and Fotini Arabazti ${ }^{2}$ \\ ${ }^{1}$ Department of Mechanical Engineering and Industrial Design, Technological Education Institute of \\ Western Macedonia, Koila, 50100 Kozani, Greece. \\ ${ }^{2}$ School of Physical Education \& Sport Science, Aristotle University of Thessaloniki, Ippokratous 22 \\ Ag. Ioannis, 62122 Serres, Greece.
}

\begin{abstract}
Several studies sought to determine impact attenuation of shoes through the evaluation of force platform or accelerometer measurements. Recent literature however points out methodological flaws of "subject inclusive" experimentation, as this approach is unable to evaluate neither the foot/ground interface nor the absorbed energy allocation. This study provides a holistic evaluation of the biomechanical response of athletic footwear to individual runner characteristics through advanced mechanical testing.
\end{abstract}

\section{INTRODUCTION}

As a periodic motion, running exposes the human musculoskeletal system to intense impacts amounting up to 2.5 times the runner's weight. The repeated nature of these impacts over long distances has been documented to induce joint overloading and a variety of other running related injuries. Cushioning systems, found in technical footwear, provide protection to recreational and professional athletes in mitigating these transient forces. Lieberman et al. [1] showed that the specific properties of these midsole systems o can change individual gait patterns. These changes are provoked by sensory mechanisms at the foot-ground interface (during foot-strike), which the body perceives as a source of potential injury. Creagh et al. [2] also suggest that the terrain plays a crucial part in the running style and observed that stride length and stance phase increases both, at higher speeds and on soft terrain.

A holistic understanding of kinematics is required to evaluate human locomotion and performance during running. When a moving object (e.g. the foot) collides with a nonmoving one (e.g. the ground), there is an exchange of energy and momentum between the 2 objects. This periodic phenomenon re-occurs during each foot-strike and results in a transient force that is transmitted, throughout our musculoskeletal system. Whittle [3] showed that the magnitude of the transient forces and the subsequent transient shock waves are highly dependent on the material between the food-ground interface, suggesting the use of viscous-elastic material for the construction of midsole systems of athletic shoes. With a

\footnotetext{
* Corresponding author, email: maropou@teiwm.gr
} 
probability of 1 injury for every 1000 hours of training [4], the shock mitigation is arguably the most important aspect of technical footwear.

The ground reaction forces recorded during running have a distinctive pattern, which recent studies have associated to both, body mass and the running speed [5]. Although in theory the more flexible the cushioning system, the less percussive the forces, impact mitigation is a non-linear phenomenon, complicating the prediction of a shoe's absorbing capacity. The ground reaction, during foot-strike, is distributed by the midsole in multiple dimensions (space, time and a subject specific frequency spectrum). As a result, the effect of the cushioning material might be concealed (to some extent) in low frequency components of the active swing phase, inducing forces to other foot parts. Furthermore, most studies [6] assume that the ground reaction force is exercised in only one direction, accelerating the center of mass, whereas gait patterns are more accurately described by multi-body systems [7].

The human body compensates for the transient forces created during the foot-ground strike through both, suitable joint alignment and the presence of viscous-elastic tissue in the heel bed and the joints. Athletic footwear provides additional protection, as its main purpose is to alter the perception of the different environmental conditions in favor of the runner [8], by dissipating the transient shock waves created during the foot-ground interface. However, individuals identify environmental stimuli and comfort in a different way, thus rendering the optimal footwear choice subjective. The choice of the type of footwear cushioning can be affected by the morphological characteristics of an individual foot [9] and other criteria that are not always bound to the shoe quality and performance.

Over the past decade several studies sought to determine impact attenuation of shoes through the evaluation of force platform or accelerometer measurements ([3], [10], [11]). Recent literature however [5] points out methodological flaws of "subject inclusive" experimentation, as the former approach is unable to evaluate neither the foot/ground interface nor the absorbed energy allocation.

Despite the abundance of different running shoes, most of the available cushioning systems can be assigned to one of three materials: foam, gel and air based. Ideally a shockabsorbing midsole, regardless of material type, should dissipate the kinetic energy of the impact, while transmitting a constant plateau force to the runner [12]. It is therefore essential to determine which cushioning system accommodates individual athletes better, in order to render running-related injuries less prevalent.

The primary hypothesis of this investigation is that the shock attenuating capacity of different midsole systems, is strongly related to impact energy and thus, each shoe type should be optimized for a specific body mass. This study provides an in-depth evaluation of the biomechanical response of athletic footwear to individual runner characteristics (weight and impact speed) through advanced mechanical testing and compares the shock absorption capacity of the three types of cushioning systems (foam, gel and air).

\section{EXPERIMENTAL METHODS}

ASTM standards suggest the evaluation of running shoes through guided impacts ranging from 5 to 7 joules (ASTM F1614-99, [13]; ASTM F1976-06, [14]). Even though it is adequate to evaluate the shock absorption capacity of athletic footwear destined for a 50 percentile male (175 $\mathrm{cm}$ height and $78 \mathrm{~kg}$ body mass) running at a high pace, it is not sufficient to cover a wide spectrum of sub-elite runners, which are expected to significantly vary in body mass and thus impact energy. According to literature, a 50-percentile male should have a shoe size of 42 [15], but anthropometric data suggest that this shoe size would also cover individuals varying by more than $\pm 30 \mathrm{~kg}$ in body mass. This indicates that 
the impact energy would exceed or fall short of the test limits by more than $40 \%$, an error that might increase even further if gender-specific criteria are considered.

As the aim of this investigation was to determine the dependence of impact attenuation properties of athletic footwear by the runner's body mass, only the conditions occurring at the end of the swing phase (foot strike) were simulated. A polymer (ABS) heel was prototyped on an open source 3D printer (MendelMax 2) to match both, the impact pattern of a classic heel-strike runner and the midsole geometry of the tested shoes. This was achieved by texturing the impact module's lower surface [5]. The shoe-heel was mounted on a guidance unit, providing rigid support to the shoe while ensuring the application of the impact load in the desired direction. The assembly consisted of two linear rails, guiding the axial ball bearings on which the heel was mounted [5]. Shock attenuation was measured using an INSTRON CEAST 9350 drop-weight testing machine.

The experimental assembly along with one shoe specimen at a time, was inserted into the device. The load, corresponding to a runner body mass range of $45-70 \mathrm{~kg}$, was propagated onto the midsole through the artificial heel. $5 \mathrm{~kg}$ impact load variations towards a $10 \mathrm{~kg}$ under- and $20 \mathrm{~kg}$ over-weight subject were also considered. Two different strike velocities were tested, 0.4 and $0.5 \mathrm{~m} / \mathrm{s}$. Additionally, the mass of the heel-guidance assembly, placed between the force transducer and the shoe, was considered during the drop test calibration to avoid interference with the force and acceleration data.

In order to achieve the body mass range of $45-70 \mathrm{~kg}$ some adjustments were made taking into consideration the different strike velocities. For $0.4 \mathrm{~m} / \mathrm{s}$ the $45-70 \mathrm{~kg}$ range translated to 3.6 to $5.8 \mathrm{~J}$ of strike energy and equivalent mass of 7.014 to $11.014 \mathrm{~kg}$, while for $0.5 \mathrm{~m} / \mathrm{s}$ the strike energy was in the range of 5.6 to $8.75 \mathrm{~J}$ and the equivalent mass was in the range of 11.014 to $17.514 \mathrm{~kg}$. Three type of cushioning systems (foam, gel and air based) were evaluated for loads corresponding to a female runner of average body mass. For each type of shoe the test was conducted 3 times, to ensure the good repeatability of the results. All specimens were new, as prior impact conditioning would significantly alter the shock attenuation capacity of footwear [5]. All the tests were conducted at $25{ }^{\circ} \mathrm{C}$ and normal humidity. Several parameters were registered during each test. The on shoe (cushioned) impact force was registered along with the maximum vertical displacement and the $\%$ force attenuation.

\section{RESULTS}

The test results for both speeds, where mild running corresponds to $0.4 \mathrm{~m} / \mathrm{s}$ and fast running to $0.5 \mathrm{~m} / \mathrm{s}$, and for three types of midsole systems are shown in Figure 1. For the gel based cushioning almost no difference was observed among the two test speeds. Foam based cushioning lead to slightly varying impact attenuation, when considering the 0.4 and 0.5 $\mathrm{m} / \mathrm{s}$ impact velocity, whereas air based cushioning exhibited rather significant differences. Specifically, for the air technology fast running produces $8 \%$ less impact force compared with mild running across the whole range of body masses examined. As for the foam cushioning fast running develops 2-3\% more force, revealing a less loading-rate dependent behavior when compared to air.

Further examination of the results reveal a strong relation of the impact force, generated during foot-strike, to the runner's body mass. This was notable for both, air and gel cushioning, while the foam technology was not greatly affected by the weight, at least at the range tested in this study. For the gel cushioning the difference in the force dissipation performance between the highest and the lowest point is $250 \mathrm{~N}$ and for the air cushioning is $140 \mathrm{~N}$. At this point it must be considered that these are impact forces that transmitted to the musculoskeletal system at every foot-strike, thus indicating the significance of footwear choice in terms of fatigue phenomena over long distance running. 


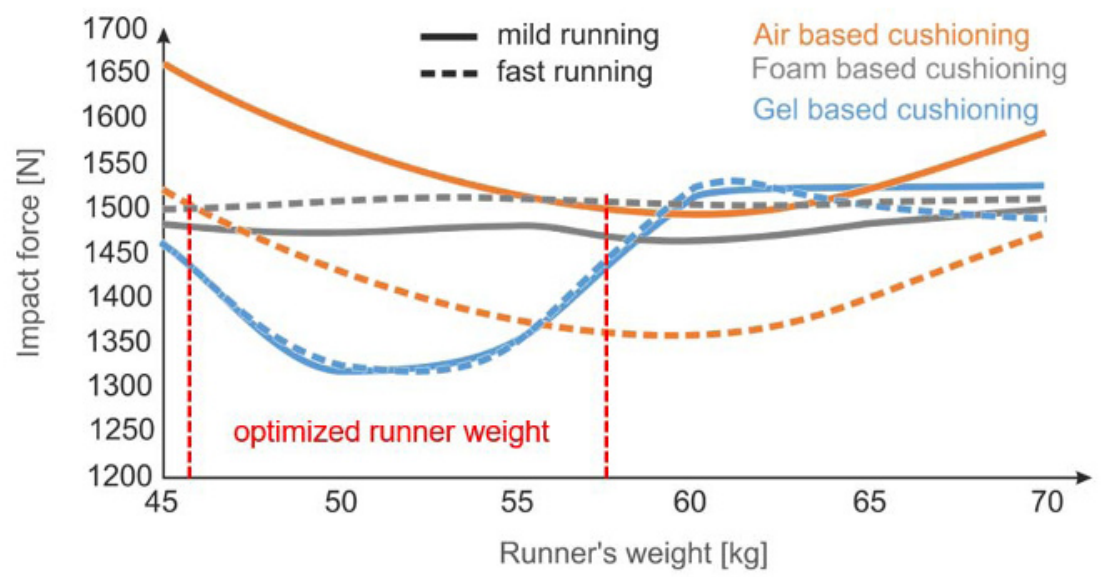

Fig. 1: Measured impact force for all types of cushioning.

Gel based cushioning exhibited a superior performance when compared to the other midsole systems, at least at a body mass range of $45-56 \mathrm{~kg}$, as is shown in Figure 1. For heavier athletes, any cushioning might be suitable depending the body mass and the type of training. Specifically, for fast running the air cushioning systems accommodates athletes in the range 56 to $70 \mathrm{~kg}$ better, as the impact force transferred to the foot is significantly lower throughout this span. However, the performance of air cushioning for mild running is the worst of all three in the whole range tested in this study, excluding the $60-65 \mathrm{~kg}$ span. For mild training the foam based technology is superior to the other two for runners with body mass from 58 to 70 , making it the best choice in this body mass range.

\section{DISCUSSION}

The investigation provided refined insight on running induced impact transmission to the human body. The results showed that the impact attenuation properties of the shoe are weight depended for the two out of three cushioning types tested in this study.

The pressure relief (responsible for the comfort) in the area of the foot is dependent of the elastic properties of the material used for the footwear midsole, while the dissipation of the transient shock wave (injury prevention) during foot strike depends on properties such as viscosity [11]. If the midsole is too elastic energy will be rebound to the foot, which will result in a higher impact force. Viscous midsoles, on the other hand, will result in lower forces, but the reduction in elasticity may slow down the shape recovery of the material [3]. This is the reason why technical footwear usually employs viscous-elastic materials as cushioning media. Viscous-elastic materials are however strain-rate dependent, a response that was affirmed by the catalogued results. The loading rate, which is strongly dependent on activity type (e.g. running will result in higher impact velocities than walking or even jogging), is considered an important factor for midsole materials. At higher strain-rates, materials are expected to display increased stress values [16], which may influence the activation of secondary absorption mechanisms, often found in cushioning structures.

The results of this investigation are in line with this. Gel based cushioning is generally the result of a composite sole material, comprising of a gel surrounded by a foam. As a result, these cushioning types absorb energy by dissipating the load though the gel phase, while evoking a secondary response once the gel is deformed beyond a threshold that forces it against the foam. This induces a bi-directional strain that is only activated at higher strain-rates, thus providing superior support during running. This contributed to the superior 
performance of the gel-based midsole, when compared to the other two cushioning systems. It should however be mentioned that this was observed for a specific loading range and rate, while other limitations include the use of just one shoe size and gender consideration.

Nonetheless, optimal footwear choice remains an important issue for millions of recreational and professional runners. According to the results of this investigation, the impact dissipation capacity of footwear systems depends on both, body mass and activity type (loading range). As a result, the choice of the athletic footwear must dependent on the anthropometric characteristics of the athlete, as a less optimal shoe will lead to increased impact loads that might prove strenuous if exposed over long distances.

\section{CONCLUSION}

The principals of momentum and energy preservation would suggest the impact and reaction force, are a product of body mass and acceleration/deceleration. In these terms, athletes with increased body mass should be exposed to higher impact forces when compared to lighter subjects.

The results of this investigation clearly state that this is not the case for the midsole cushioning systems tested. Runners with lower body mass may subject to significantly increased impact forces, comparing to heavier ones, as a midsole may be underperforming if out of its optimal range.

Although modern footwear is capable of properly attenuating the shock occurring during foot strike, athletic shoes should be carefully chosen in order to avoid muscle and joint related injuries.

\section{REFERENCES}

1. D. E. Lieberman, M. Venkadesan, W. A. Werbel, A. I. Daoud, S. D'Andrea, I. S. Davis, R. O. Manq'eni, Pitsiladis, Nature, 463, 531-535, (2010)

2. U. Creagh, T.Reilly, A. Lees, Ergonomics, 41, 7 ,1029-1033, (1998)

3. M. W. Whittle, Gait and Posture, 264-275, (1999)

4. W. van Mechelen, 14, 5, 320-335, (1992)

5. A. Tsouknidas, M. Pantazopoulos, D. Sagris, D. Fasnakis, S. Maropoulos, F. Arabatzi, N. Michailidis, article ID 7136238, (2017)

6. M. R. Shorten, International conference on the Engineering of Sport, (2002)

7. N. Pandey, S. Roshan, R. Kharate, M. Sonawane, V. Bhivate, N. S. Ujwal, 3, 5, 66-70, (2014)

8. J. J. Gibson, Houghton Mifflin, (1979)

9. M. Kouchi, M. Mochimaru, H. Nogawa, and S. Ujihashi, Paper presented at the 7th Footwear Biomechanics Symposium, (2005)

10. M. F. Bobbert, H. C. Schamhardt, B. M. Nigg, J. Biomechanics, 24, 1095-1105, (1992)

11. H. A. Gruber, K. A. Boyer, T. R. Derrick, J. Hamill, Journal of Sport and Health Science, 113-121, (2014)

12. R. A. McNeil, American Scientist, 72, (1984)

13. ASTM F1614-99, 8 Applied Bionics and Biomechanics ASTM International, (2006), https://www.astm.org

14. ASTM F1976-06, ASTM International, (2006), https://www.astm.org

15. R. H. Miller, B. R. Umberger, J. Hamill, G. E. Caldwell, Proceedings of the Royal Society, 1498-1505, (2012)

16. K.D. Bouzakis, G. Maliaris, A. Tsouknidas, Computational Materials Science, 59, 133 139 (2012). 